COMPRENDRE

\title{
La diffusion Raman exaltée de surface
}

\author{
Leïla BOUBEKEUR-LECAQUE ${ }^{1}$, Nordin FELIDJ \\ Marc LAMY DE LA CHAPELLE \\ ${ }^{1}$ Univ. Paris Diderot, Sorbonne Paris Cité, Laboratoire ITODYS, UMR 7086 \\ CNRS, 15 rue J.-A. de Baiff, 75205 Paris Cedex 13, France \\ ${ }^{2}$ Institut des Molécules et Matériaux du Mans (IMMM - UMR6283), \\ Université du Mans, avenue Olivier Messiaen, 72085 Le Mans Cedex 9 \\ marc.lamydelachapelle@univ-lemans.fr
}

La spectroscopie Raman est une spectroscopie vibrationnelle très peu sensible qui limite l'analyse d'espèces chimiques aux fortes concentrations. Néanmoins, lorsque des molécules sont placées au voisinage d'une surface métallique nanostructurée, il est possible d'exalter considérablement leur signature Raman. On parle alors de diffusion Raman exaltée de surface. Les remarquables potentialités de cette technique ont nourri de nombreux champs d'étude tant pour le design de substrats dits SERS-actifs, que pour l'exploration d'applications en médecine, pharmacologie, défense ou le monde de l'art.

\section{Diffusion Raman exaltée de surface : principe et mécanismes}

La spectroscopie Raman permet d'accéder aux niveaux vibrationnels des molécules (tout comme la spectroscopie infra-rouge) et par conséquent d'identifier la composition chimique et la structure de systèmes moléculaires de façon certaine. D'un point de vue physique, l'onde électromagnétique provenant d'une source laser monochromatique induit un moment dipolaire oscillant dans la molécule (le moment dipolaire induit $\mathbf{P}$ est lié au tenseur polarisabilité $[a]$ de la molécule et au vecteur champ électrique incident $\mathbf{E}$, selon : $\mathbf{P}=[a] \mathbf{E}$ ).

Si cette spectroscopie présente de nombreux avantages comme par exemple de pouvoir travailler avec des échantillons sous différents états physiques (solide, liquide ou gaz), elle présente un inconvénient majeur, à savoir que le spectre obtenu, pour des échantillons à faible concentration, est de faible intensité et peut être complètement masqué par sa fluorescence. La diffusion Raman exaltée de surface (DRES) ou SERS en anglais (surface enhanced Raman scattering) permet de surmonter cette limitation en amplifiant le signal Raman [1]. La méthode consiste à exploiter une surface métallique nano-structurée (principalement en $\mathrm{Au}, \mathrm{Ag}$ ou $\mathrm{Cu}$ ) pour exalter de plusieurs ordres de grandeurs l'intensité de la réponse Raman des molécules présentes au voisinage de la surface métallique (voir le schéma de principe sur la figure 1). Il est en effet établi que l'effet SERS trouve principalement son origine dans un phénomène d'amplification $\mathrm{du}$ champ électromagnétique diffusé par les particules métalliques (ce mécanisme est dit électromagnétique

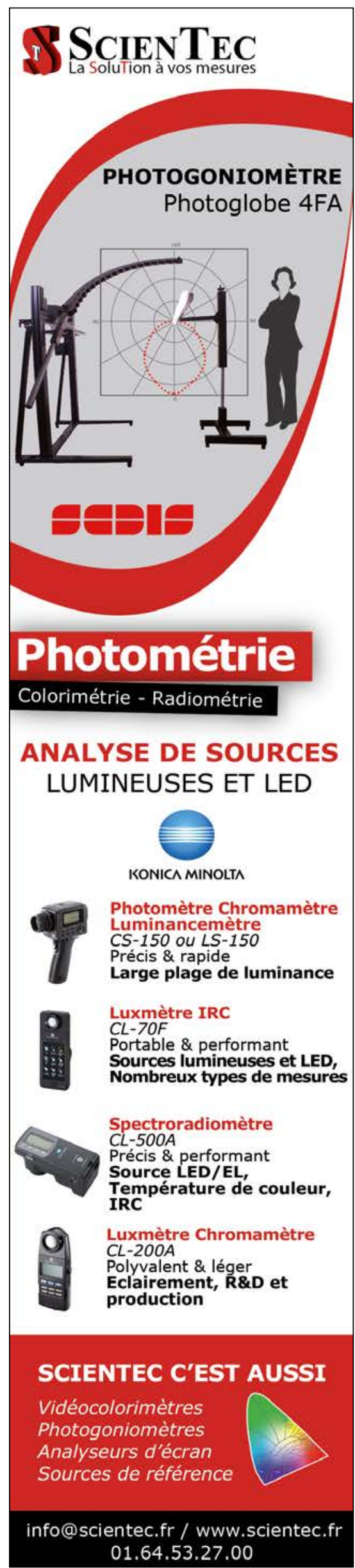




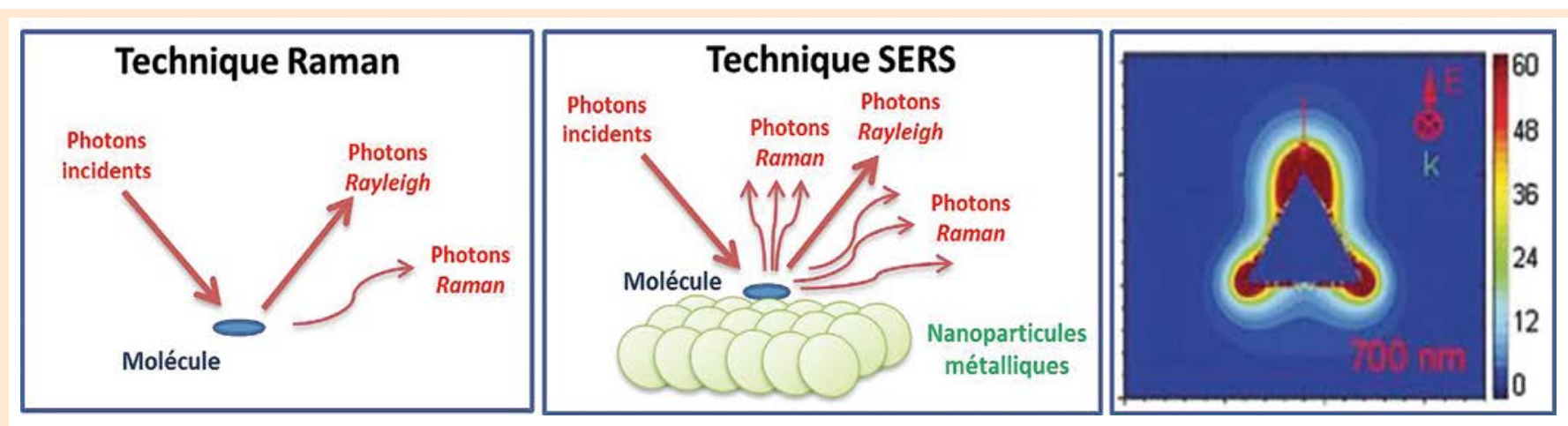

Figure 1. Schéma de principe de l'effet SERS : diffusion Raman classique (gauche); diffusion Raman exaltée de surface (centre) ; cartographie du champ électrique local pour une particule triangulaire en argent (excitation à $700 \mathrm{~nm}$ ).

car lié à l'exaltation locale du champ proche). Cette amplification est associée à l'excitation de plasmon de surface localisé ( $\left.\mathrm{PSL}^{1}\right)$ sur des nanoparticules métalliques. En effet, ces dernières présentent des résonances optiques, qui «s'étalent» depuis le proche UV (ultra-violet) $(350 \mathrm{~nm}$ pour l'argent) jusqu'au proche IR (visible et proche IR pour l'Au).

Ces résonances se traduisent par l'apparition de bandes d'absorption et de diffusion très intenses en champ lointain, dont la longueur d'onde PSL dépendra principalement de la nature chimique des nanoparticules, de leur taille, de leur forme, de leur espacement ou de l'indice de réfraction du milieu environnant. En champ proche, le phénomène PSL se caractérise par une amplification considérable du champ électrique local $\mathbf{E}_{\text {loc }}$ à la longueur d'onde de la résonance PSL (de 2 ordres de grandeur dans le meilleur des cas), ce qui génère l'exaltation du moment dipolaire induit Raman, et donc des spectres Raman des molécules présentes au voisinage de la surface des particules (figure 1). Il est important de noter que l'effet SERS perd de son efficacité dès lors que la molécule se trouve au-delà d'une dizaine de nanomètres de la surface de la particule, en raison de la décroissance exponentielle du champ électrique en fonction de la distance. Ainsi, le facteur d'exaltation, proportionnel à la puissance quatrième du champ électrique local $\mathbf{E}_{\text {loc }}$, peut atteindre des valeurs de l'ordre de $10^{7}-10^{8}$.

Il existe également un autre mécanisme d'exaltation d'origine moléculaire, qui se traduit par une augmentation de la polarisabilité de la molécule par transfert de charges résonant entre le métal et l'adsorbat. Il a cependant été montré que ce mécanisme génère, dans les conditions les plus favorables, des gains de l'intensité Raman de l'ordre de $10^{2}$ qui viennent alors s'ajouter à l'exaltation en $\mathrm{E}^{4}$ liée à l'augmentation du champ électromagnétique local.

Par conséquent, le phénomène SERS met en jeu nécessairement trois partenaires : la lumière, une molécule et une surface métallique (aussi appelée substrat SERS) avec des PSL excitables par la lumière. Un des enjeux majeurs en SERS concerne la recherche des substrats SERS-actifs, présentant un maximum de gain Raman pour la caractérisation spectrale d'objets moléculaires à l'état de traces (systèmes biologiques, molécules toxiques, drogues...) [3].

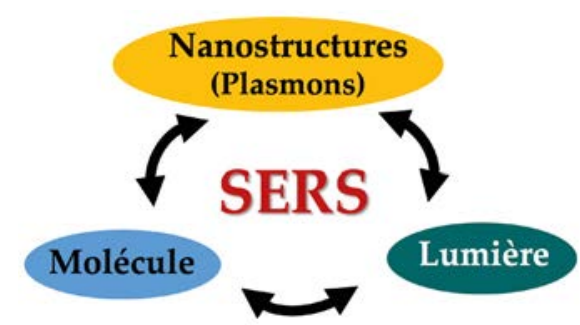

Les PSL correspondent à des oscillations collectives des électrons de conduction à la surface du métal sous l'effet d'un rayonnement électromagnétique incident.

\section{Les substrats SERS}

Généralement, les substrats SERS sont des nanostructures à base de métaux dont la fréquence de résonance PSL se situe dans la même gamme que les fréquences d'excitation Raman (du visible au proche-IR). L'or et l'argent sont de loin les métaux les plus utilisés pour les substrats. Bien que potentiellement très efficaces et élargissant le domaine spectral du plasmon à l'UV, le cuivre et l'aluminium demeurent peu utilisés du fait de la difficulté à les manipuler à l'air et à maintenir leurs performances SERS stables dans le temps.

L'élaboration de substrats présentant le maximum d'exaltation est depuis une quinzaine d'années un domaine de recherches extrêmement actif qui a abouti au développement de techniques de nanofabrication très originales et a permis des avancées majeures en synthèse de nanomatériaux métalliques. L'élan de recherches est aujourd'hui également motivé par le besoin de disposer de substrats SERS robustes, fiables et reproductibles.

Nous avons vu précédemment que la taille caractéristique nanométrique des structures est à l'origine du phénomène d'exaltation mais c'est bien la présence de points chauds qui explique les exaltations remarquables en SERS $\left(10^{7}-10^{8}\right)$. La génération de ces points chauds (zone générant une très forte exaltation) est un élément clé dans la fabrication des substrats qui peut se faire aussi bien par des stratégies ascendantes (bottom-up) que descendantes (top-down). Chaque approche 
a bien sûr ses avantages et ses inconvénients qu'il est important d'avoir à l'esprit selon l'échelle d'utilisation et l'application visée in fine.

Les substrats SERS appartiennent à l'une des catégories listées ci-après (figure 2) :

- Surfaces lithographiées : elles sont obtenues par des techniques de lithographie physique (gravure par faisceau d'ions ou d'électrons par exemple) permettant d'obtenir des nanostructures contrôlées et d'ajuster finement la position de la résonance PSL. Cependant, leur fabrication est coûteuse (temps, équipements lourds, salle blanche...) et difficilement industrialisable à grande échelle.

- Surfaces rugosifiées : elles peuvent être obtenues par exemple par des cycles d'oxydo-réduction répétés un grand nombre de fois sur une surface métallique macroscopique. Le substrat obtenu présente généralement des facteurs d'exaltation très élevés mais les réponses SERS sont inhomogènes du fait de la répartition intrinsèquement aléatoire des points chauds.

- Suspension colloïdale de particules isolées : les nanostructures correspondantes sont obtenues par voie chimique (peu coûteuse) par réduction d'un sel métallique en solution en présence d'un stabilisant (tensio-actif) de surface. Les avancées de ces 15 dernières années ont permis d'accéder à une incroyable diversité de formes de nanoparticules (sphère, cube, bâtonnet, bipyramide, oursin, étoile...) avec une amélioration substantielle de l'homogénéité de la taille des nanoparticules. La grande variété de structures (forme, taille, nature du métal) accessibles permet d'ajuster aisément la position de la résonance PSL. Néanmoins, la modification chimique de la surface des nanoparticules, l'ajout d'autres molécules, la modification du $\mathrm{pH}$ ou le changement de solvant ne sont pas toujours compatibles avec le maintien de la stabilité colloïdale. La déstabilisation de la solution conduit alors à l'agrégation irréversible de la suspension la rendant parfois inutilisable.
- Colloïdes agrégés : ils résultent de l'assemblage de plusieurs nanoparticules pour donner des agrégats riches en points chauds localisés dans l'espace étroit entre deux particules. Bien que présentant de forts facteurs d'exaltation, le contrôle du nombre de particules par agrégat est très difficile et leur faible stabilité en solution dans le temps les rend peu fiables pour la quantification analytique.

\section{Applications de la spectroscopie Raman exaltée de surface}

Comme il a été expliqué précédemment, la spectroscopie Raman exaltée de surface permet une exaltation du signal Raman des molécules situées à proximité de la surface des nanoparticules métalliques. Il est alors possible d'exploiter cette exaltation locale en vue de l'utilisation de cette technique dans plusieurs applications.

La plus évidente concerne la détection de molécules. En effet, l'exaltation étant très importante (plusieurs ordres de grandeur), il est possible d'observer une très faible quantité de molécules et donc de réduire considérablement la concentration de molécules observées. Dans certains cas quelques centaines de molécules suffisent à créer un signal Raman significatif et sous certaines conditions, il est même possible de mesurer le spectre Raman d'une molécule individuelle (single-molecule SERS). La technique SERS est donc extrêmement sensible. De nombreux capteurs basés sur le SERS ont ainsi été proposés pour la détection d'espèces chimiques et biologiques [4]. Les limites de détection en termes de concentration peuvent aller du nanomole jusqu'au femtomole par litre, ce qui est extrêmement compétitif dans le domaine des capteurs chimiques ou biologiques. De nombreuses applications ont été proposées comme la détection de polluants, d'explosifs, de contaminants alimentaires ou de biomarqueurs de maladie dans des milieux biologiques.

\section{FORMATION CONTINUE}

\author{
Une nécessité \\ pour les entreprises \\ Une mission majeure
de l'Institut d'Optique
}

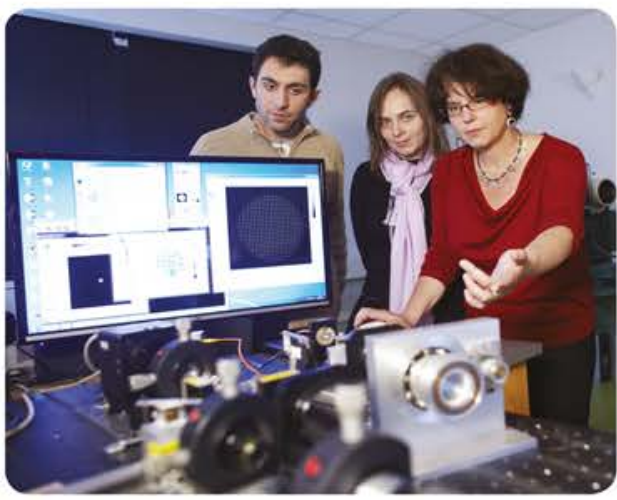

- Au catalogue ou sur-mesure

- Plus de 150 montages pédagogiques

- De l'initiation à la spécialisation

- Formateurs experts

- En anglais ou en français

- En France ou à l'étranger

- Liens forts avec l'industrie

- Infrarouge, optronique

Instrumentation, composants,

fabrication

Conception optique

Imagerie, mesure, communication

Sources, éclairage, lasers, sécurité

- Mastère Spécialisé ${ }^{\otimes}$ ELS

Systèmes d'Éclairage Embarqué

\section{Nous contacter}

Emilie Ericher

+33164533236

fc@institutoptique.fr 


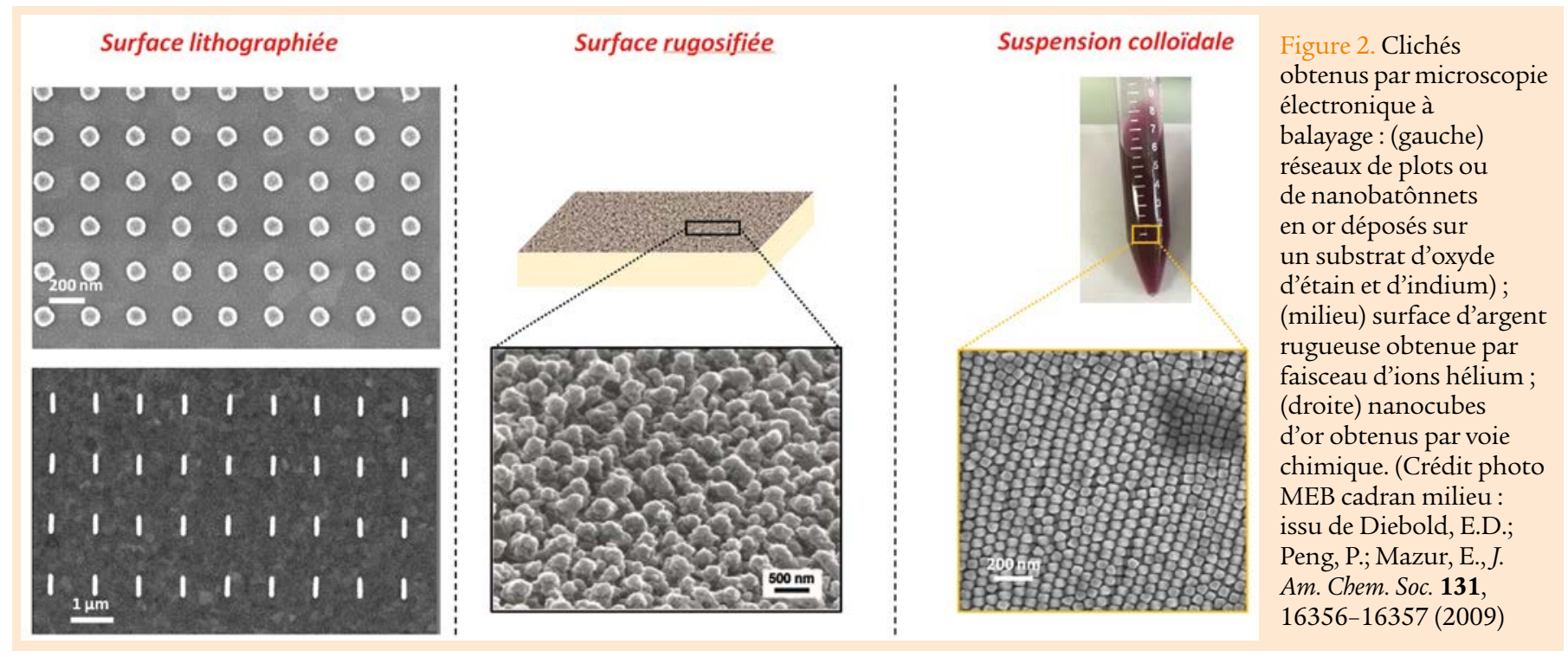

Une deuxième application est le suivi par SERS de la signature spectrale des molécules au cours de processus physico-chimiques (réaction, transfert de proton ou d'électron, photo-isomérisation, changement conformationnel...). En effet, le spectre Raman est directement relié à la nature chimique et à la structure de la molécule. Il est donc possible à partir du spectre Raman d'étudier la structure des molécules et éventuellement les modifications induites sur celle-ci suite à une interaction avec une autre molécule ou bien à un changement de l'environnement [5]. Les nanostructures servent alors de sondes pour observer les molécules à une certaine position. Ainsi il a été proposé d'injecter des nanoparticules colloïdales dans des cellules pour étudier leurs différents constituants comme les protéines ou certains composants comme les exosomes ou les lysosomes. Il a également été proposé d'utiliser les variations spectrales de certaines molécules déposées à la surface des nanoparticules pour mesurer le pH localement à l'intérieur de la cellule.

Dans le même esprit, il est également possible d'observer des modifications de la structure d'une molécule suite à une réaction chimique, ce qui permet de suivre les différents produits lors de la réaction. Ceci est d'autant plus pertinent que le PSL qui permet l'exaltation locale du champ électromagnétique et par conséquent du signal Raman peut aussi être utilisé pour induire des réactions chimiques. C'est ce qu'on appelle la plasmonique moléculaire. Dans ce cadre, le SERS peut donc être utilisé pour suivre les réactions induites par plasmon [6].

Une dernière application est la mesure du champ proche. En effet, la molécule dont le signal Raman est exalté est localisée dans le champ proche de la nanoparticule. La molécule peut alors être utilisée comme sonde de l'exaltation du champ proche. Il est donc possible d'estimer cette exaltation ainsi que son extension spatiale (longueur de décroissance) en fonction des paramètres géométriques de la nanoparticule.

Le SERS est en conclusion une technique très puissante pour l'observation et l'étude des molécules. Il permet notamment le développement de dispositifs concrets s'appuyant sur l'identification et la quantification ultra-sensible de molécules. La maîtrise des méthodes de production des échantillons ainsi que leur exploitation pour de nombreuses applications spécifiques en font une méthode optique incontournable pour la caractérisation des systèmes moléculaires.

\section{POUR EN SAVOIR PLUS}

[1] N. Félidj, J. Aubard, G. Lévi, J.R. Krenn, A. Hohenau, G. Schider, A. Leitner, F.R. Aussenegg, Optimized surface-enhanced Raman scattering on gold nanoparticle arrays, Appl. Phys. Lett. 82, 3095 (2003)

[2] E. Hao, G. C. Schatz, Electromagnetic fields around silver nanoparticles and dimers, J. Phys. Chem. 120, 357-366 (2004)

[3] Haidar, I.; Levi, G.; Mouton, L.; Aubard, J.; Lau-Truong, S.; Grand, J.; Neuville, D.R.; Felidj, N.; Boubekeur-Lecaque, L., Highly stable silica-coated gold nanorods dimers for solution-based SERS, Phys. Chem. Chem. Phys. 18, 32272 (2016)

[4] N. Guillot, M. Lamy de la Chapelle, Lithographied nanostructures as nanosensors, Journal of Nanophotonics 6, 64506 (2012)

[5] M. Cottat, R. Yasukuni, Y. Homma, N. Lidgi-Guigui, N. Varin-Blank, M. Lamy de la Chapelle, C. Le Roy, Phosphorylation impact on spleen tyrosine kinase conformation by surface enhanced Raman spectroscopy, Scientific Reports 7, 39766 (2017)

[6] I. Tijunelyte, E. Guenin, N. Lidgi-Guigui, F. Colas, J. Ibrahim, T. Toury, M. Lamy de la Chapelle, Nanoplasmonics tuned "click chemistry", Nanoscale 7, 7105 (2016) 